\title{
Integrating hybrid runoff generation mechanism into Variable Infiltration Capacity model to facilitate hydrological simulation
}

\author{
Youjiang Shen ${ }^{1}$, Dedi Liu ${ }^{1}$, Jiabo Yin ${ }^{2}$, Lihua Xiong ${ }^{3}$, and Pan Liu ${ }^{1}$ \\ ${ }^{1}$ State Key Laboratory of Water Resources and Hydropower Engineering Science \\ ${ }^{2}$ Stat Key Laboratory of Water Resources and Hydropower Engineering Science \\ ${ }^{3}$ State Key Lab of Water Resources and Hydropower Engineering Science
}

April 28, 2020

\begin{abstract}
The runoff generating processes and water fluxes exchange are essential simulating the physical nonlinear rainfall-runoff relations in hydrological models. However, current runoff generation representation in hydrological models is constraint due to both vertical and horizontal heterogeneity of meteorological factors and underlying surface condition, hampering the streamflow simulation performances. To address this concern, by integrating a hybrid runoff generation mechanism in the upper layer and baseflow curve in the lower layer, this work develops a modified Variable Infiltration Capacity (VIC) hydrological model. With consideration of both vertical and horizontal heterogeneity runoff, the modified VIC model can simulate Horton and Dunne runoff through the hybrid mechanism and lateral runoff based on three soil-layer structure. The upper reach of Hanjiang River basin with substantial climate variations is selected as a case study to validate the performance of the modified model. Results show that the modified VIC model can produce a better performance of streamflow simulation in terms of Nash-Sutcliffe Efficiency, at around 0.8 on average, especially for semi-humid and mountainous catchments. In addition, parameters related to the hybrid mechanism are sensitive to the hydrological performance, indicating that the improved performance should be attributed to the hybrid mechanism.
\end{abstract}

\section{Hosted file}

Main_text.docx available at https ://authorea.com/users/309948/articles/440807-integrating-hybridrunoff-generation-mechanism-into-variable-infiltration-capacity-model-to-facilitate-hydrologicalsimulation 


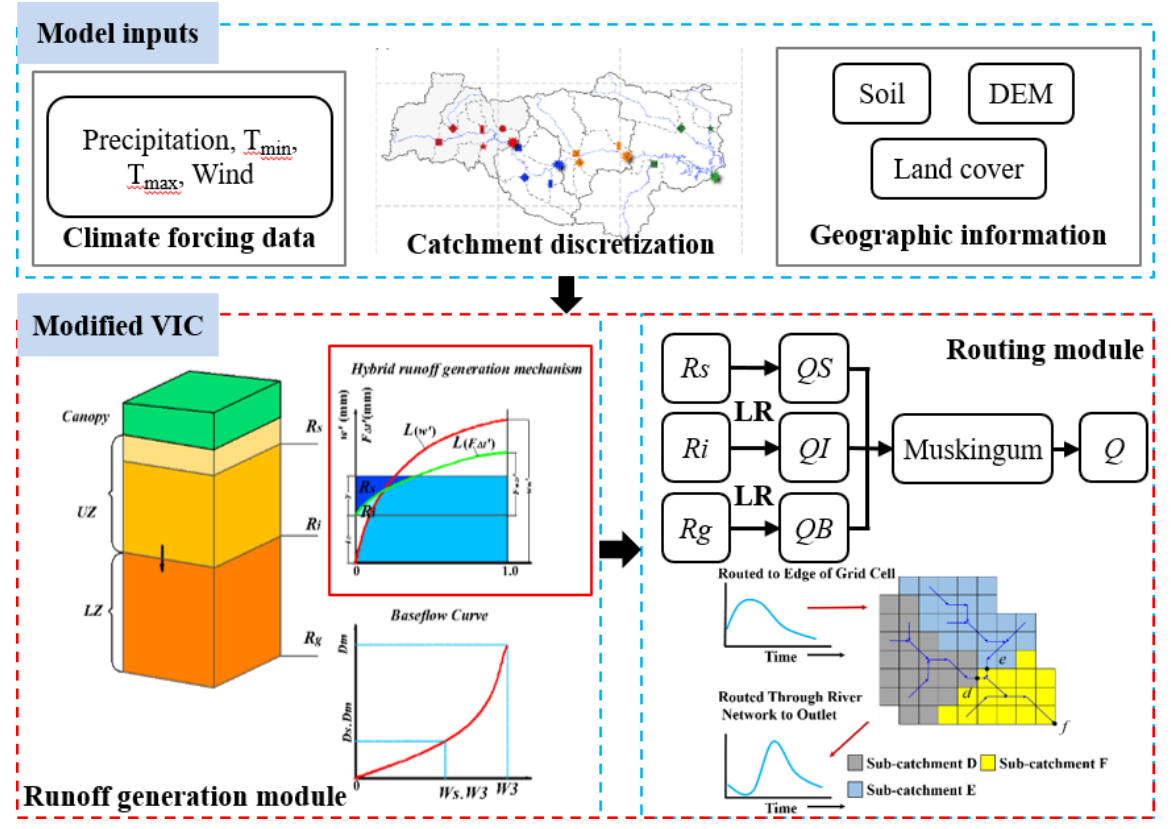

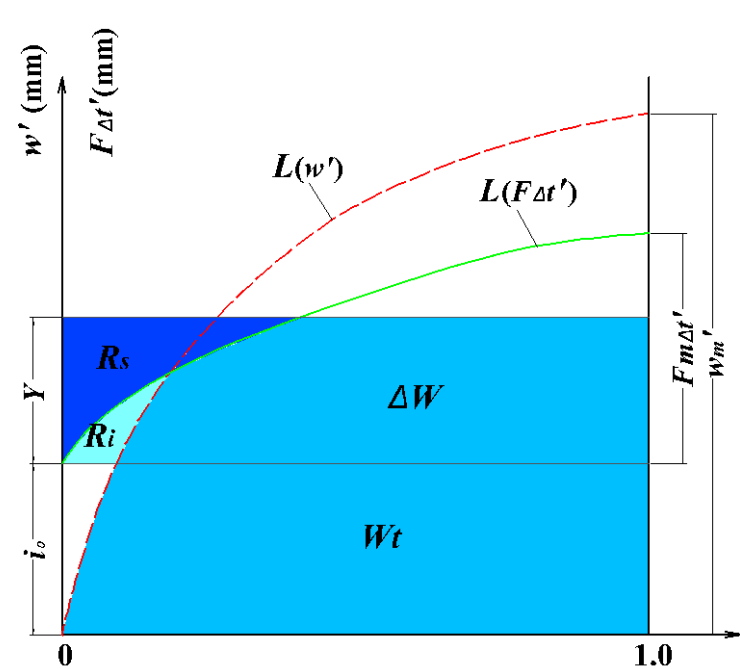

(a)

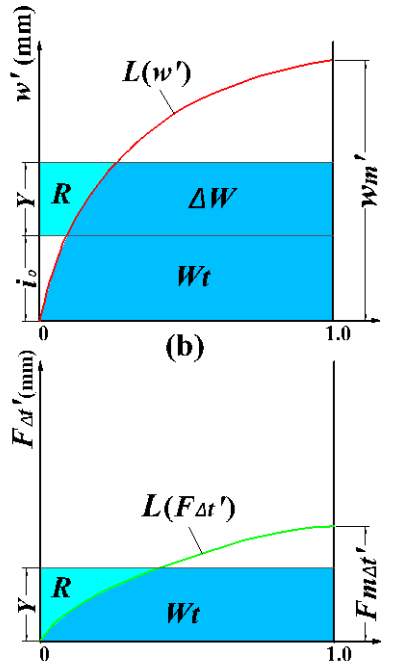

(c) 


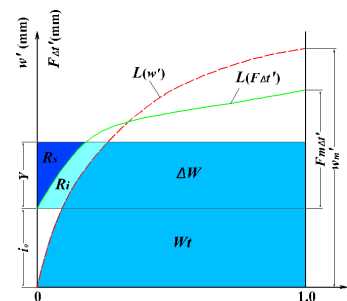

(a)

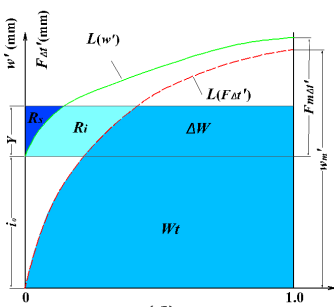

(d)

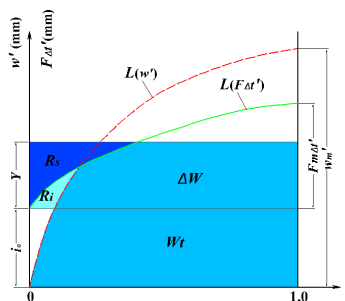

(b)

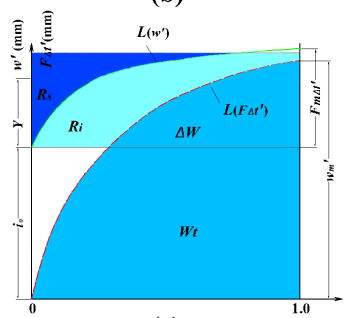

(e)

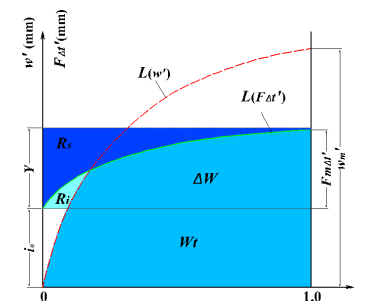

(c)

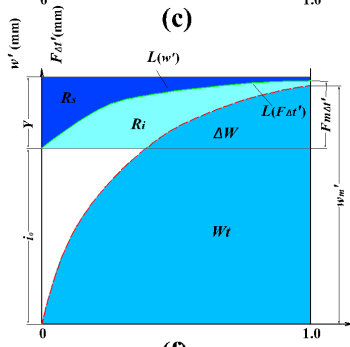

(f)
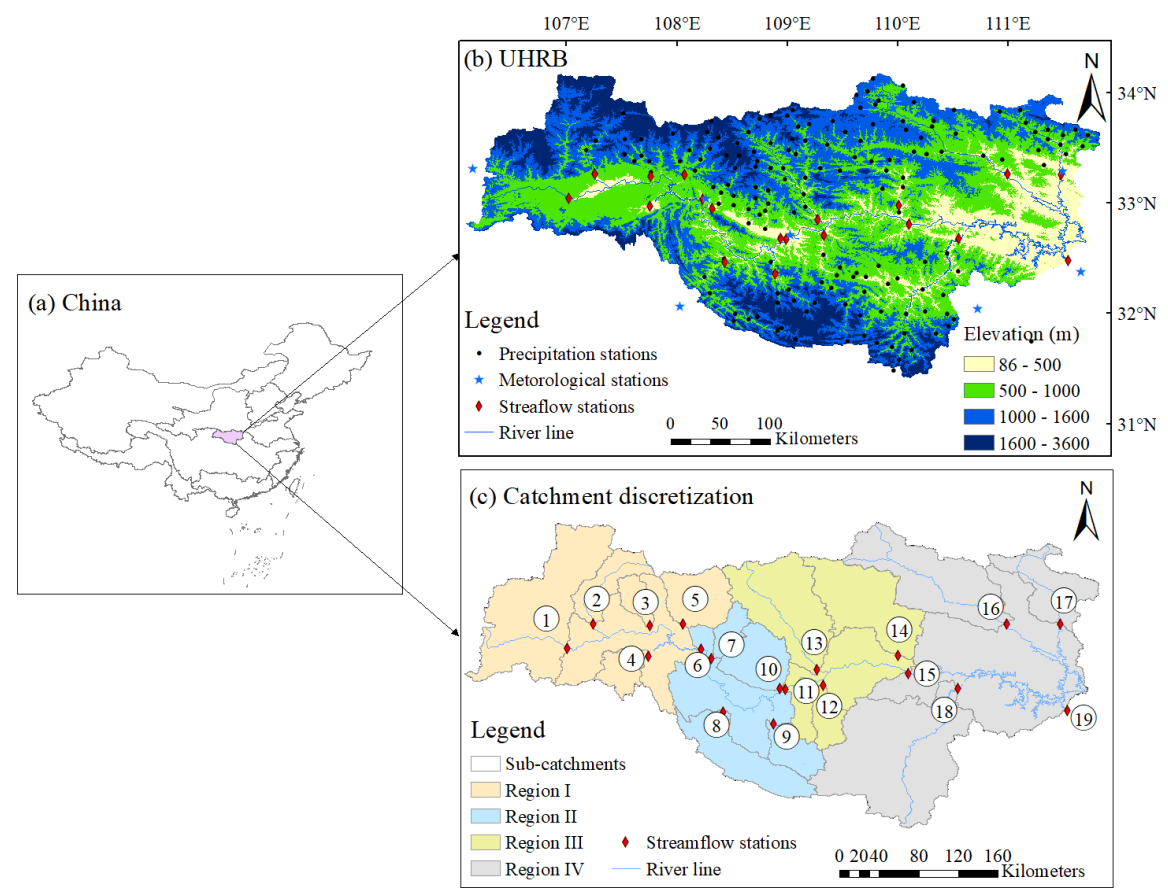

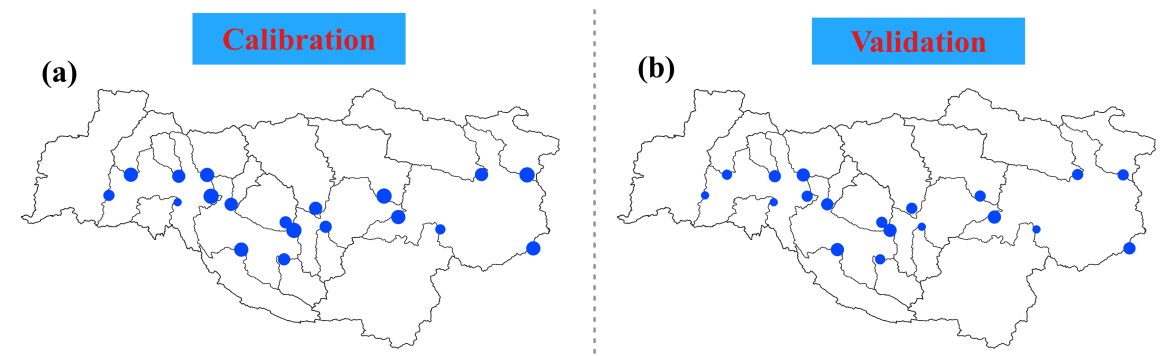

(c)

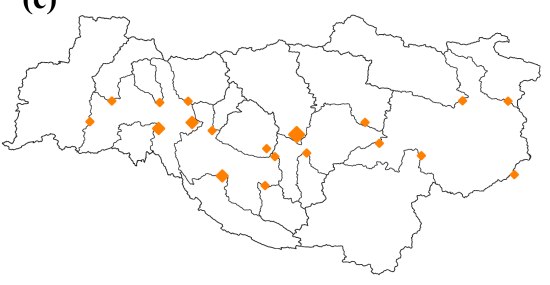

(d)
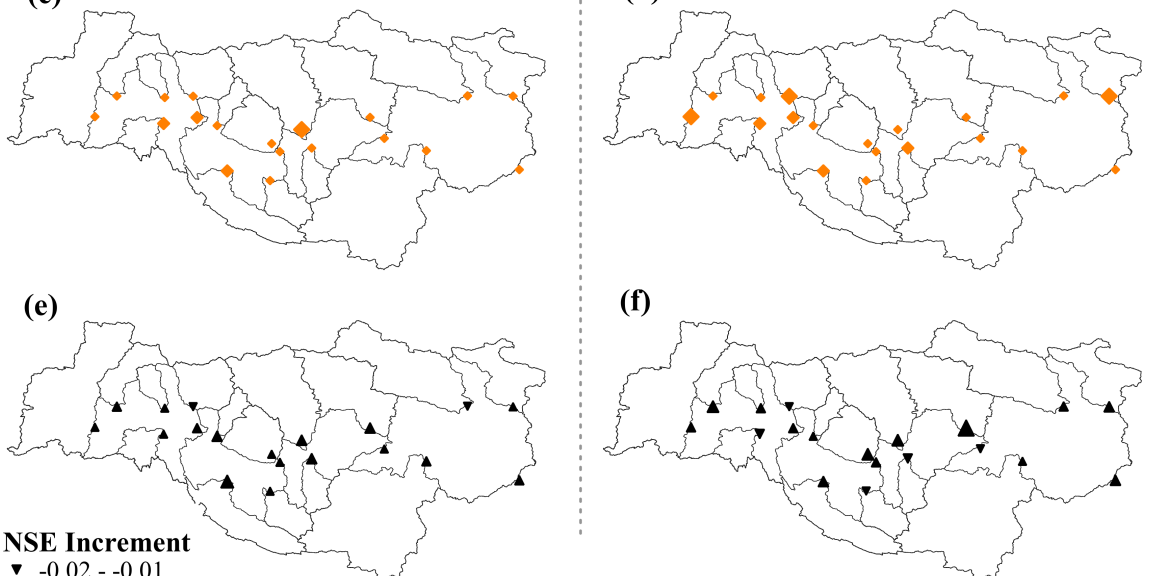

(f)

$\checkmark-0.02--0.01$

- $-0.01-0.00$

- $0.00-0.01$

- $0.01-0.02$

$\triangle 0.02-0.03$

\ $0.03-0.04$

$\Delta \quad 0.04-0.05$

А $0.05-0.06$

$\Delta$ 0.06-0.07

А $0.07-0.08$

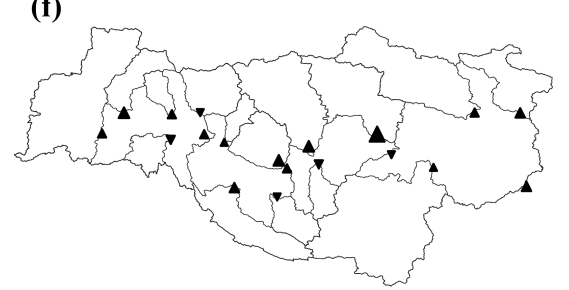

NSE

- $0.59-0.73$

- $0.73-0.76$

- $0.76-0.79$

- $0.79-0.82$

- $0.82-0.85$

- $0.85-0.88$

- $0.88-0.91$

RE

$-30.0--20.0$

$-20.0--10.0$

- $-10.0-0.0$

- $0.0-10.0$

$-10.0-20.0$

- $20.0-30.0$
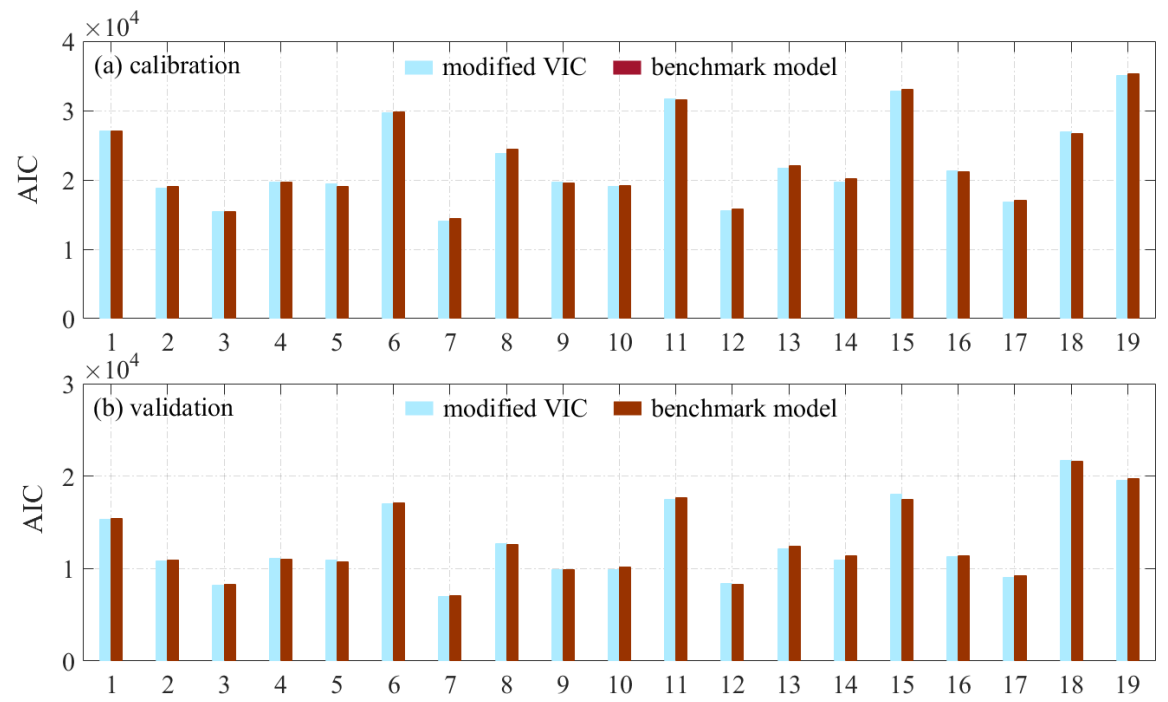

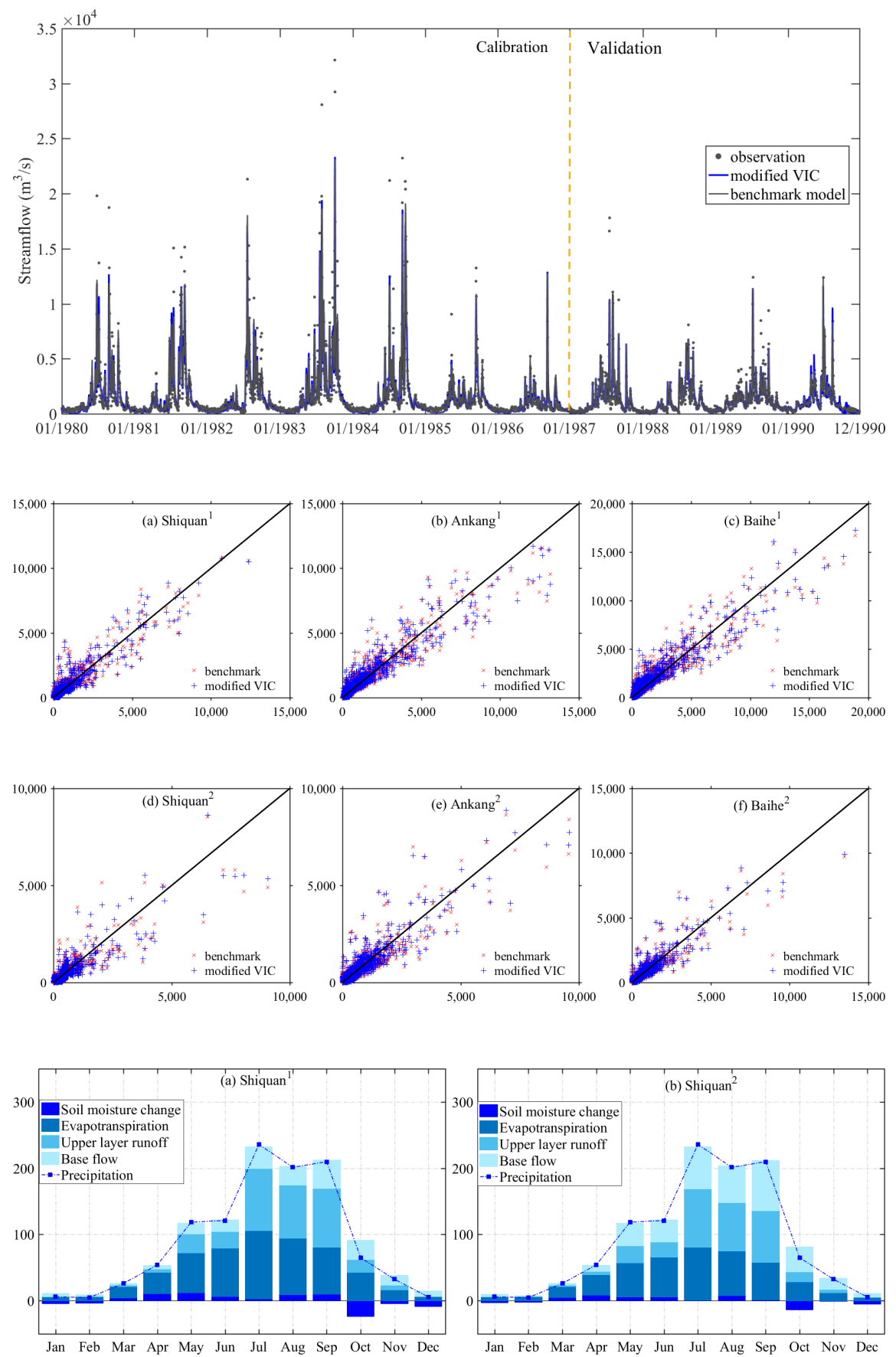

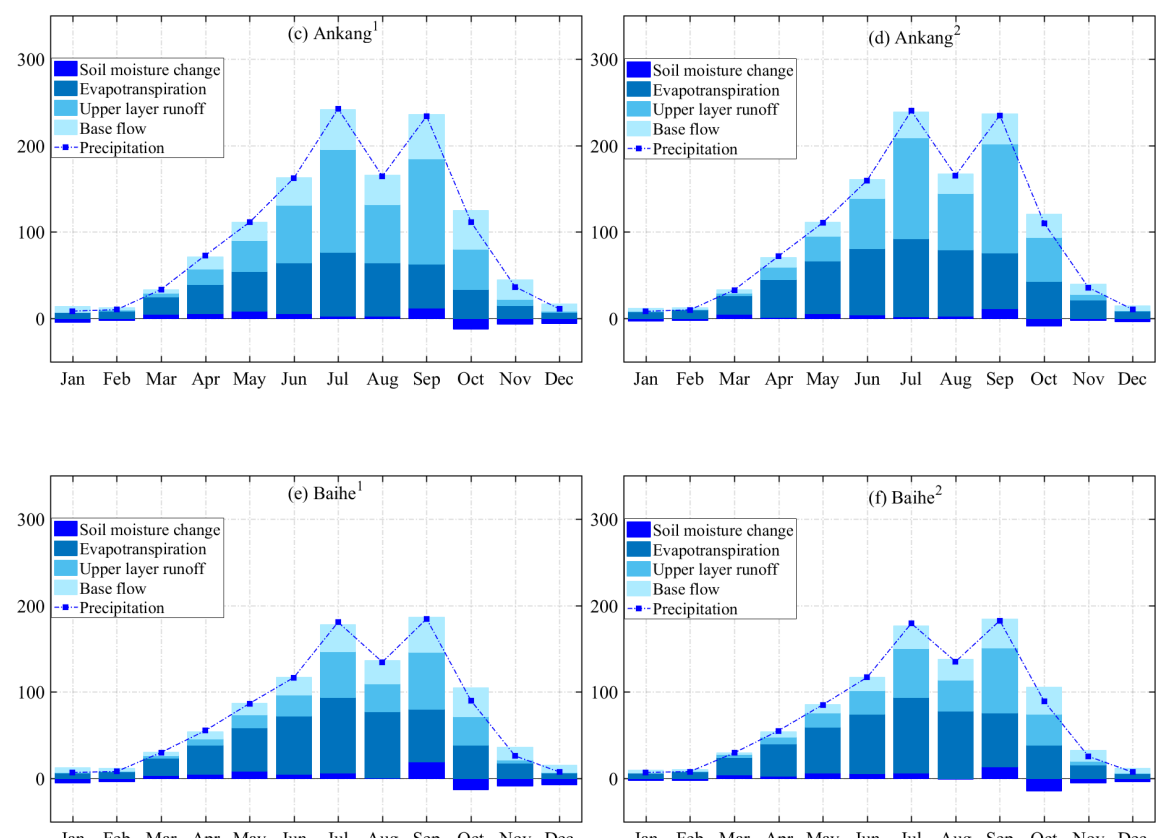

Jan Feb Mar Apr May Jun Jul Aug Sep Oct Nov Dec

Jan Feb Mar Apr May Jun Jul Aug Sep Oct Nov Dec
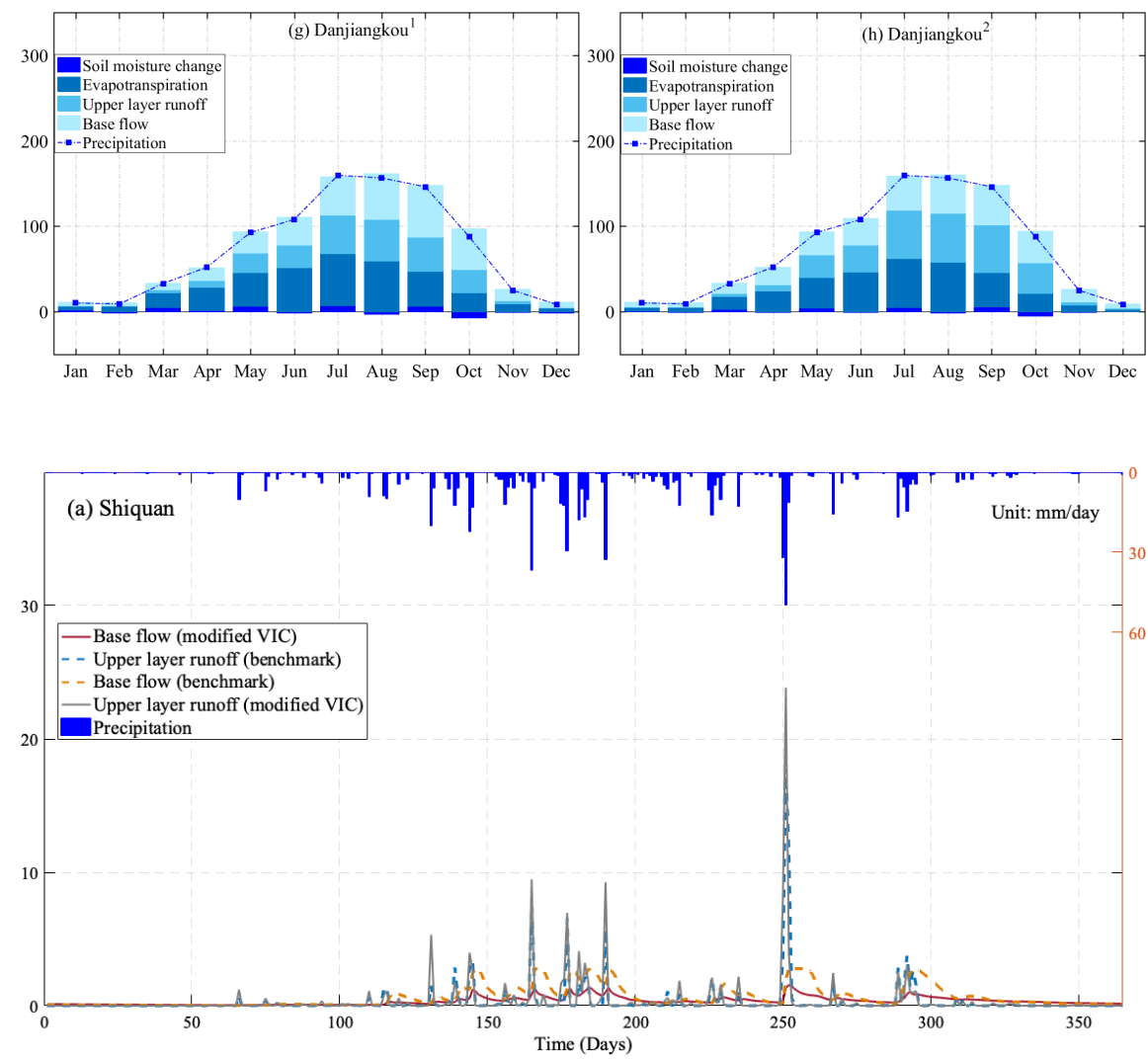

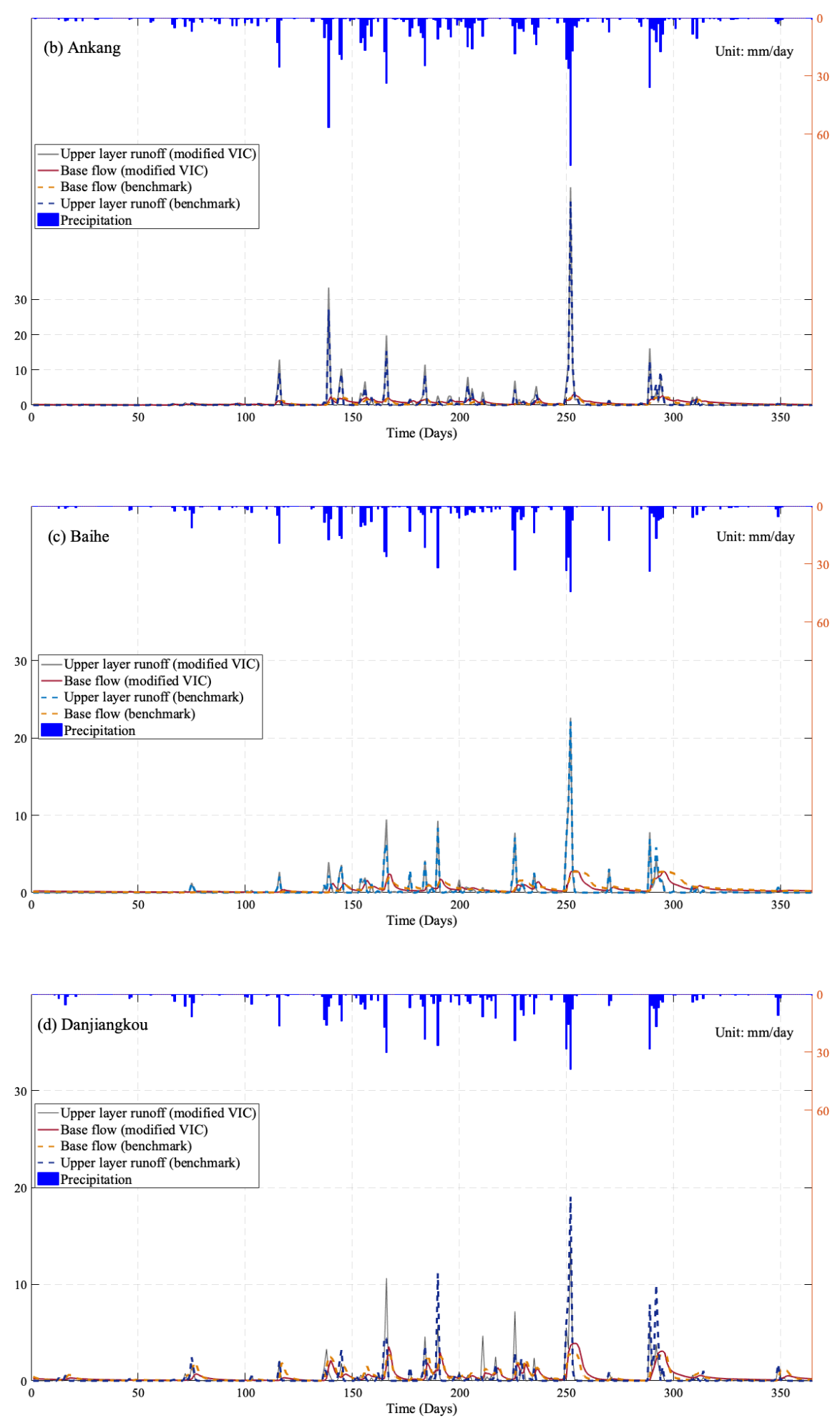

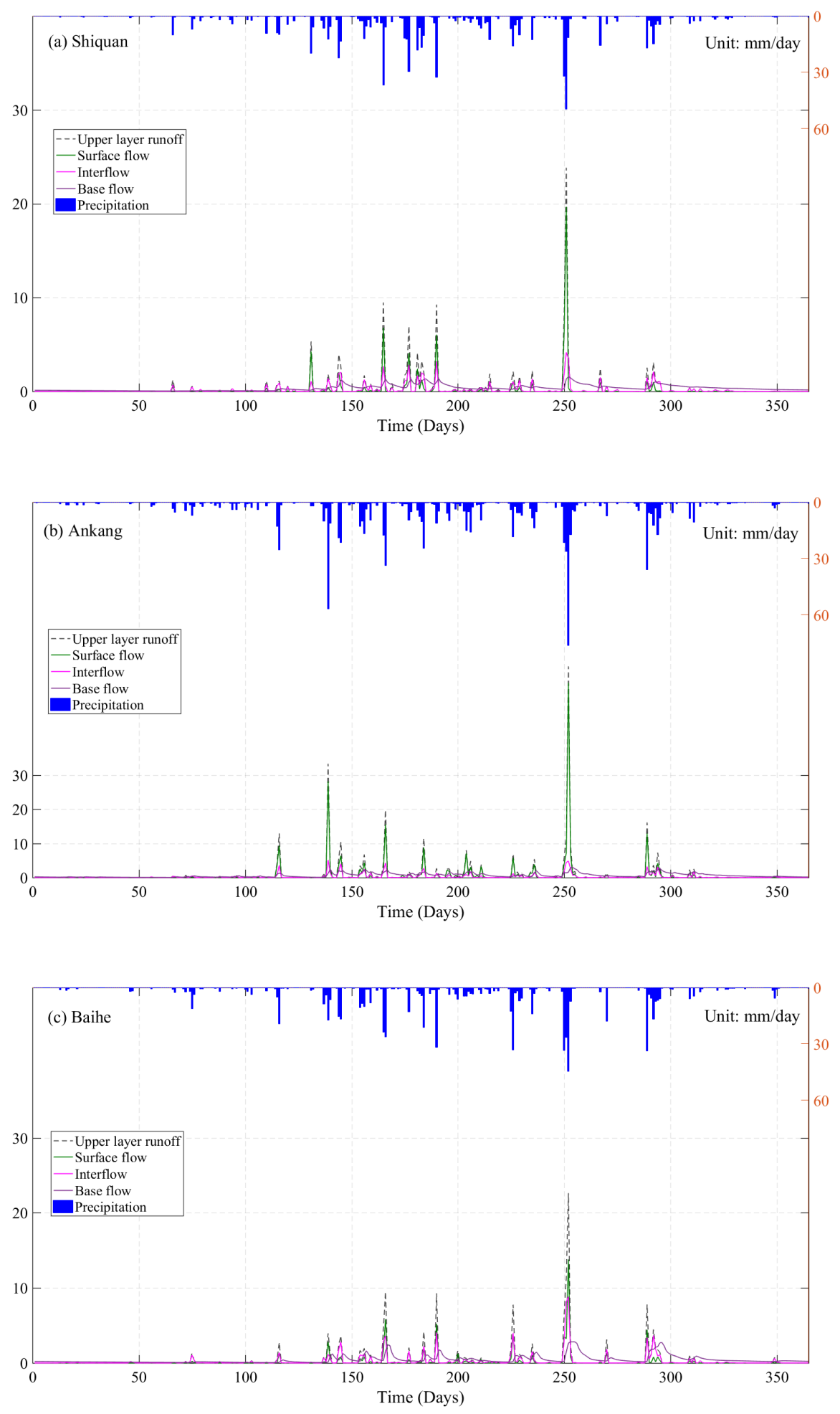

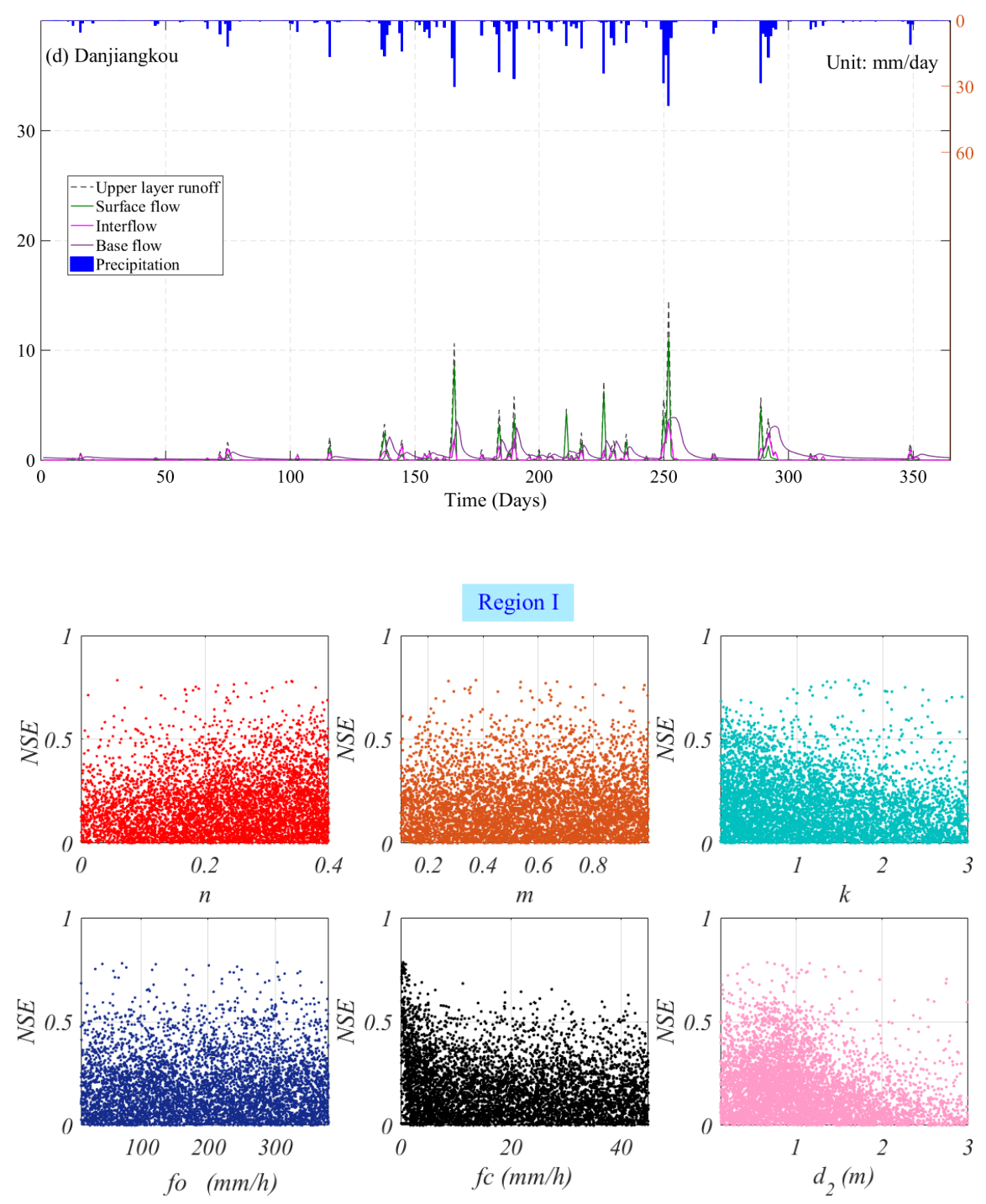

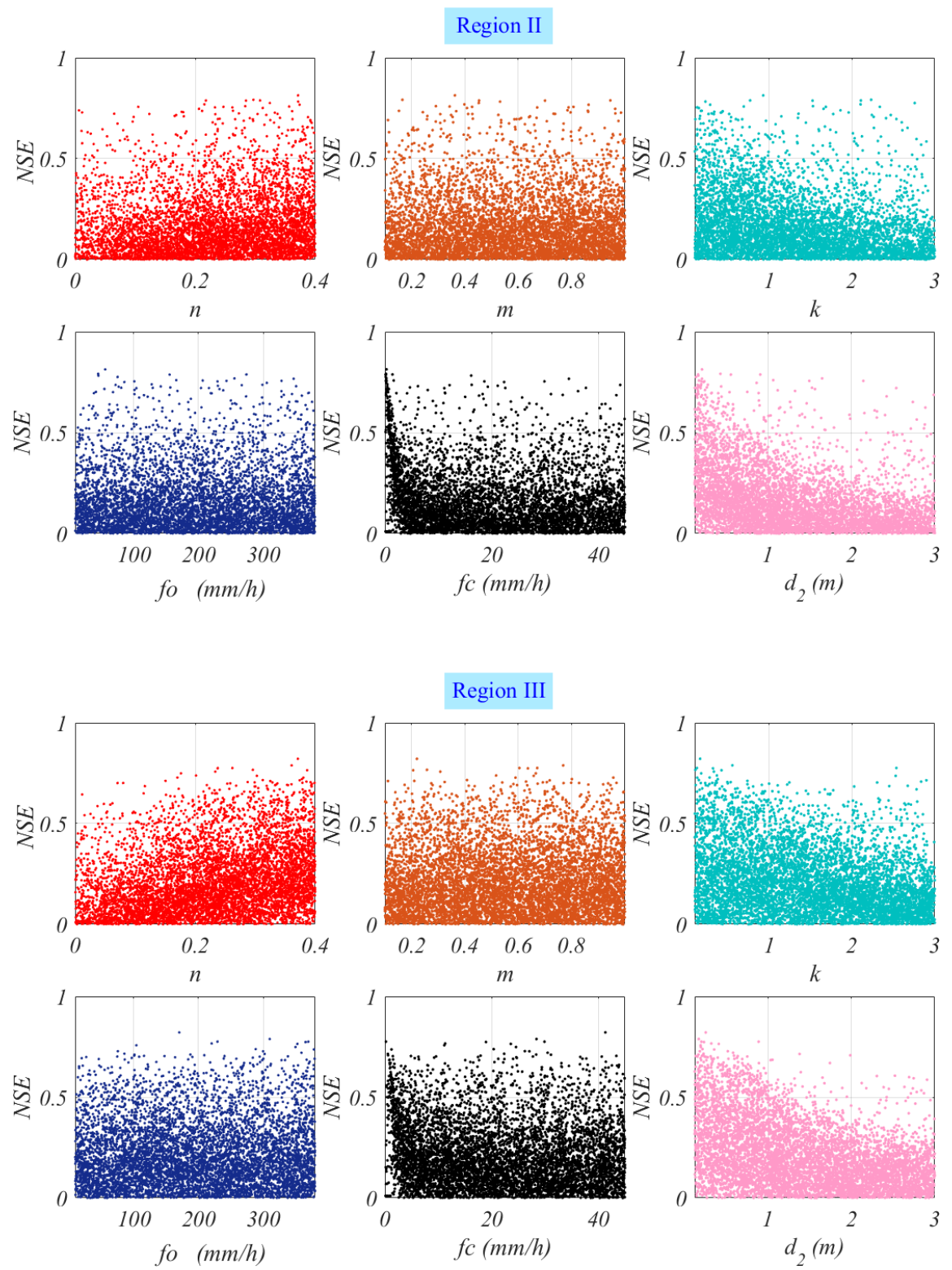

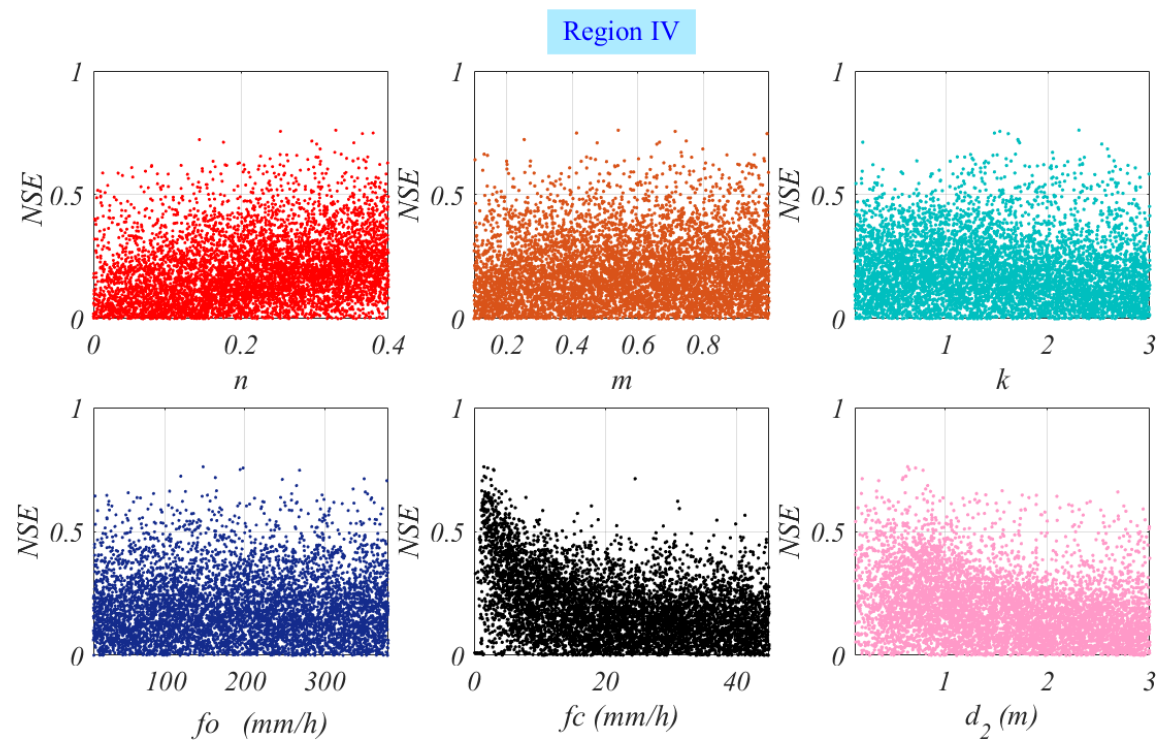